\title{
3D extended field-of-view of the knee joint bones using ultrasound: Application in orthopaedics
}

\author{
Maged Nasan ${ }^{1,2}$, Yannick Morvan ${ }^{3}$, Guillaume Dardenne ${ }^{2,4}$, Jean Chaoui ${ }^{3}$, \\ Eric Stindel ${ }^{1,4}$ \\ ${ }^{1}$ University of Western Brittany, Brest, France \\ ${ }^{2}$ LaTIM, Brest, France \\ ${ }^{3}$ Wright Medical, Plouzané, France \\ ${ }^{4}$ Brest University Hospital Center, Brest, France \\ nasan@univ-brest.fr
}

\begin{abstract}
Patient Specific Instruments (PSIs) have been introduced into the surgical workflow as a modern way to assist the surgeon in performing femur and tibia resection in Total Knee Arthroplasty (TKA). These PSIs are based on an accurate reconstruction of the surface of the knee's bones.

In this work, we propose two 3D-3D image-based registration methods to reconstruct an extended field-of-view of the knee joint using only a motorized ultrasound transducer. Those methods are: (1) a dense voxel-based registration method, which needs to preprocess the ultrasound images and form an ultrasound volume. Then, computing the Mutual Information (MI) for each relative displacement to align every pair of volumes, (2) a sparse point-based registration method, which takes into account the point set located on the surface of the bone in ultrasound images. This method detects bony features using ORB detector and matches the corresponding points to find the best transformation using Coherent Point Drift (CPD).

The preliminary qualitative results performed in vitro show that from a set of consecutive ultrasound volumes, an extended field-of-view can be reconstructed using only ultrasound images without any external trackers. Results of the voxel-based approach show that MI is more robust against noise comparing to other similarity measures. On the other hand, results of point-based approach show that is much faster in computation with a low false-positive rate compared to other feature-detectors like SIFT and SURF. Furthermore, experiments show that CPD is less affected by noisy data compared to the classical ICP, which is promising to continue evaluating our work in vivo.
\end{abstract}




\section{Introduction}

Accurate bone resections are critical to properly perform a Total Knee Arthroplasty (TKA) (Nam 2012). Enable to assist the surgeon in performing these cuts, Patient Specific Instruments (PSIs) have been introduced into the surgical workflow. These PSIs are typically based on a 3D surface reconstruction of the distal femur and the proximal tibia extracted from pre-operative MRI or CT scanners (Mattei 2016). The idea followed is to design and 3D print a personalized instrument for which the shape corresponds to the negative of the bones shape. In the case of a TKA, the surgeon can simply place a femur PSI and a tibia PSI onto the distal femur and proximal tibia since the shape of the two PSIs perfectly fit the shape of the bones. A major disadvantage of these CT- or MRI-based PSIs is that they require expensive and time-consuming pre-operative scanners.

In this work, we propose a novel solution that enables the reconstruction of the distal femur and proximal tibia surfaces using ultrasound images, thereby tremendously simplifying the design of PSIs. The idea followed is to reconstruct an extended field of view of the bone surfaces by combining multiple smaller ultrasound volumes (Poon 2006). The major benefits of this method are that it does not require markers or localizers and can be performed by the surgeon during the patient examination.

\section{Materials and Methods}

All images are acquired by a motorized transducer connected to an ultrasound system (Ultrasonix SonixTABLET). We perform several acquisitions of the knee joint from different poses, every acquisition giving a stack of 2D B-Mode frames. We propose to use image-based 3D-3D registration to find the best geometrical transformation that aligns every two consecutive volumes. Hence, we get an extended field-of-view of the knee. In this work, we investigated two different approaches to perform the registration of two consecutive ultrasound volumes:

(1) Dense voxel-based registration method and (2) a sparse point-based registration method. The two approaches are explained below.

For dense voxel-based, we need to preprocess the data before the alignment. Starting from a stack of 2D frames, we need to scan-convert the images. Scan-conversion is a geometric transformation that gives the fan shape to a series of parallel ultrasound frames. However, the gaps (zero-voxels) between frames resulting from the scan-conversion form a noise that cannot be neglected. To avoid forming this noise, a pixel-wise-mean frame can be inserted between every two adjacent frames before the scanconversion. We call this technique frame interpolation, which can be repeated several times for one stack of frames. Finally, we perform the scan-conversion process. Once we have an interpolated scanconverted volume out of each stack of frames, we perform a rigid 3D-3D voxel-based registration using MI similarity measure of each pair of consecutive volumes.

Sparse point-based registration, on the other hand, is a different approach to find the best geometric transformation of two consecutive poses of the echo probe. Only a set of 3D points located on the surface of the bone is taken into account instead of the entire ultrasound volume. We assume that the bone surface in all ultrasound volumes is a rigid surface. However, the main challenge here is to isolate the bone surface from the noise and the other soft tissues. Removing the noise in this step is essential because the noise affects dramatically the point matching step that comes later. Therefore, to build a point set that represents the bone surface out of a stack of frames: First, bone surfaces are automatically extracted using local phase image features (Hacihaliloglu 2008) of each 2D frame. Then, we extract the Oriented FAST and rotated BRIEF (ORB) features (Rublee 2011), which represent the points on the bone surface. Finally, we scan-convert the extracted points to correct their coordinates in 3D. Once we get a geometrically-corrected point set of each volume, we perform the so-called Coherent Point Drift 
(CPD) (Myronenko 2010) point matching approach to find the best alignment of every two consecutive point sets.

\section{Results}

The preliminary qualitative results performed in vitro show that from a set of consecutive ultrasound volumes we can construct an extended field-of-view using only ultrasound images without any tracking markers as shown in Figure 1 and Figure 2.

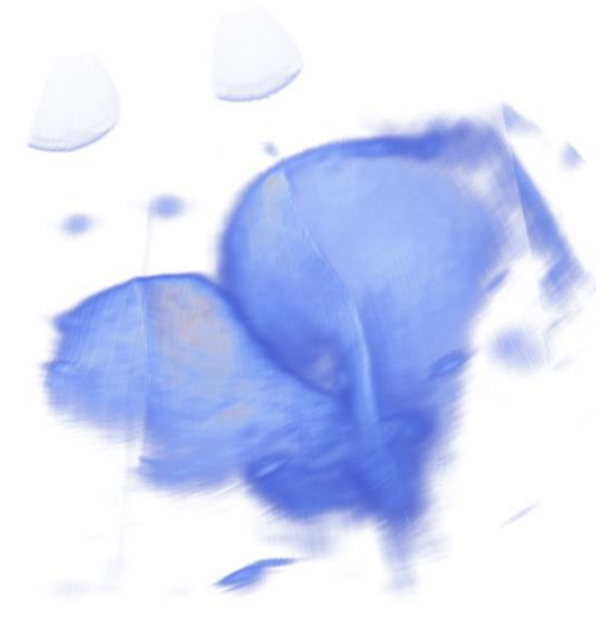

Figure 1: Pair of ultrasound volumes aligned using the dense voxel-based approach. The part in the middle is a shared region between the two, while the left part belongs to the first volume and the right part belongs to the second

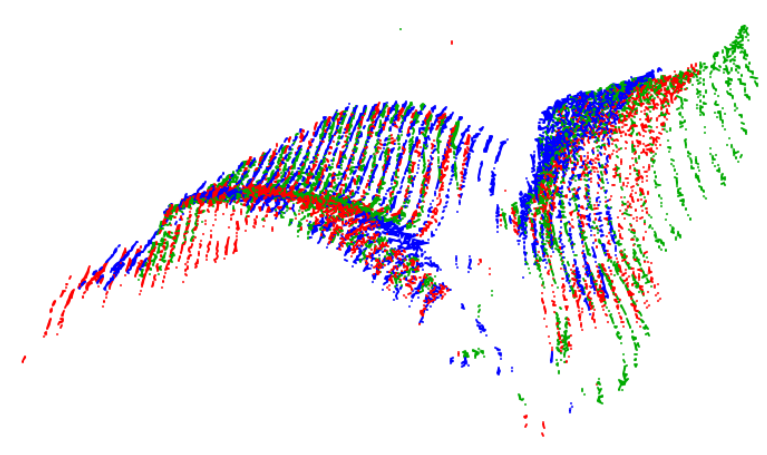

Figure 2: Alignment of three ultrasound volumes of knee joint bones using the point-based approach. The points of each volume are given a different color (red, blue and green) 


\section{Discussion}

The main objective of this work is to study the ability to construct the 3D surface of the distal femur and proximal tibia using only ultrasound data.

In dense voxel-based approach, several methods were tested including different similarity measures (Wachinger 2007) for performing the rigid registration such as Mean Squares and Normalized Correlation with and without interpolation. Experiments show that frame interpolation technique is crucial to avoid local minima during the computation of the similarity measure, while results show that MI is more robust against the noise represented by moving soft tissue and speckle.

Concerning the point-based approach, results show that bone surface detection using ORB features is faster in term of computation with fewer false positives when comparing with 3D SIFT (Ni 2009) and SURF features. While CPD is less affected by noise compared with classical point matching methods like Iterative Closest Point (ICP).

Future work will focus on the in-vitro quantitative evaluation of the reconstruction and the in-vivo validation of the bone surface reconstruction.

\section{References}

Nam, D. (2012). Patient-specific instrumentation in total knee arthroplasty: a review. In The journal of knee surgery 25.03: 213-220.

Mattei, L. (2016). Patient specific instrumentation in total knee arthroplasty: a state of the art. In the Annals of translational medicine 4.7.

Poon, Tony C. (2006). Three-dimensional extended field-of-view ultrasound. In The Ultrasound in medicine \& biology 32.3: 357-369.

Hacihaliloglu, I. (2008). Bone Segmentation and Fracture Detection in Ultrasound Using 3D Local Phase Features. In International Conference on Medical Image Computing and Computer-Assisted

Intervention. Springer, Berlin, Heidelberg: 287-295

Rublee, E. (2011) ORB: An efficient alternative to SIFT or SURF. In Computer Vision (ICCV), 2011 IEEE international conference on. IEEE.

Myronenko, A. (2010). Point set registration: Coherent point drift. In IEEE transactions on pattern analysis and machine intelligence 32.12: 2262-2275.

Wachinger, C. (2007). Three-dimensional ultrasound mosaicing. In the International Conference on Medical Image Computing and Computer-Assisted Intervention. Springer, Berlin, Heidelberg.

$\mathrm{Ni}$, D. (2009). Reconstruction of volumetric ultrasound panorama based on improved 3D SIFT. In Computerized Medical Imaging and Graphics 33.7: 559-566. 